\title{
The size effect in metal cutting
}

\author{
MILTON C SHAW \\ Emeritus Professor of Engineering, Arizona State University, Tempe AZ, \\ 85287-6106, USA
}

\begin{abstract}
When metal is removed by machining there is substantial increase in the specific energy required with decrease in chip size. It is generally believed this is due to the fact that all metals contain defects (grain boundaries, missing and impurity atoms, etc.), and when the size of the material removed decreases, the probability of encountering a stress-reducing defect decreases. Since the shear stress and strain in metal cutting is unusually high, discontinuous microcracks usually form on the metal-cutting shear plane. If the material being cut is very brittle, or the compressive stress on the shear plane is relatively low, microcracks grow into gross cracks giving rise to discontinuous chip formation. When discontinuous microcracks form on the shear plane they weld and reform as strain proceeds, thus joining the transport of dislocations in accounting for the total slip of the shear plane. In the presence of a contaminant, such as $\mathrm{CCl}_{4}$ vapour at a low cutting speed, the rewelding of microcracks decreases, resulting in decrease in the cutting force required for chip formation. A number of special experiments are described in the paper that support the transport of microcracks across the shear plane, and the important role compressive stress plays on the shear plane. Relatively recently, an alternative explanation for the size effect in cutting was provided based on the premise that shear stress increases with increase in strain rate. When an attempt is made to apply this to metal cutting by Dinesh et al (2001) it is assumed in the analysis that the von Mises criterion pertains to the shear plane. This is inconsistent with the experimental findings of Merchant. Until this difficulty is taken care of, together with the promised experimental verification of the strain rate approach, it should be assumed that the strain rate effect may be responsible for some notion of the size effect in metal cutting. However, based on the many experiments discussed here, it is very unlikely that it is totally responsible for the size effect in metal cutting as inferred in Dinesh et al (2001).
\end{abstract}

Dinesh D, Swaminathan S, Chandrasekar S, Farris T N 2001 Proc. ASME-IMECE pp 1-8

Keywords. Structural defects; compressive stress on the shear plane; transport of microcracks.

\section{Introduction}

It has been known for a very long time that a size effect exists in metal cutting, where the specific energy increases with decrease in deformation size. Backer et al (1952) performed a 


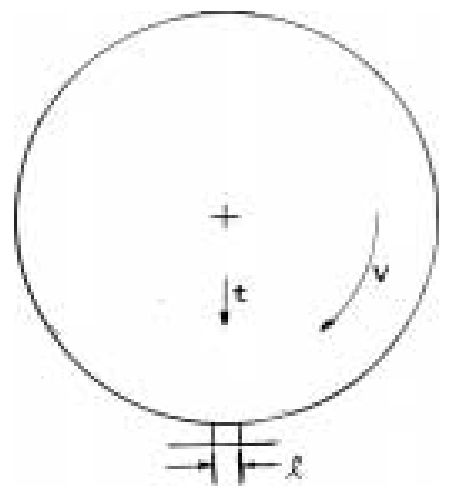

Figure 1. Plunge grinding cut with wheel engaging complete area of work with downfeed $t$ and wheel speed $V$ (after Backer et al 1952).

special series of tests in which the shear energy per unit volume deformed $\left(u_{S}\right)$ was determined as a function of specimen size for a ductile metal (SAE 1112 steel). The deformation processes involved were as follows, listed from top to bottom with increasing size of specimen deformed:

- surface grinding

- micromilling

- turning

- tensile test

Surface grinding tests were performed under relatively mild conditions involving plunge type tests in which an eight-inch $(20.3 \mathrm{~cm})$ diameter wheel was fed radially downward against a square specimen of length and width $0.5 \mathrm{in} .(1.27 \mathrm{~cm})$, as shown in figure 1 . The width of the wheel was sufficient to grind the entire surface of the specimen at different downfeed rates $(t)$. The vertical and horizontal forces were measured by a dynameter supporting the workpiece. This enabled the specific energy $\left(u_{S}\right)$ and the shear stress on the shear plane $(\tau)$ to be obtained for different values of undeformed chip thickness $(t)$ as shown in figure 2 . The points corresponding to a constant specific energy below a value of downfeed of about $28 \mu$ in. $(0.7 \mu \mathrm{m})$ are on a horizontal line due to constant theoretical strength of the material being reached when the value of $t$ goes below about $28 \mu \mathrm{in} .(0.7 \mu \mathrm{m})$. The reasoning in support of this conclusion is presented by Backer et al (1952).

In the micromilling tests a carefully balanced $6 \mathrm{in} .(152 \mathrm{~mm})$ carbide-tipped milling cutter was used with all but one of the teeth relieved so that it operated as a fly milling cutter.

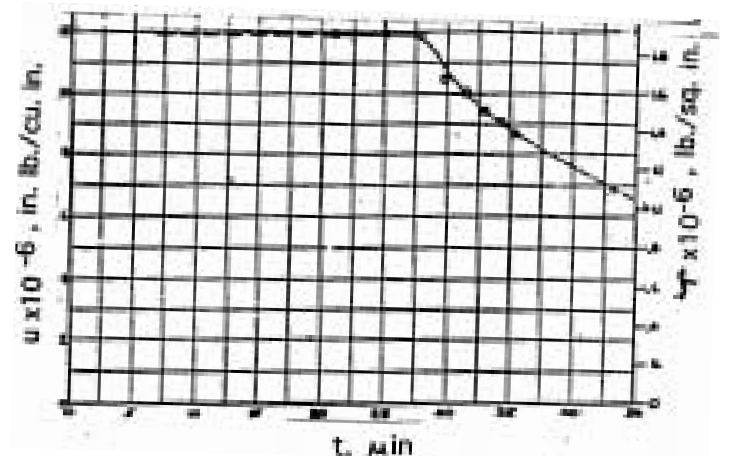

Figure 2. Specific energy-depth of cut curve for grinding tests (after Backer et al 1952). 


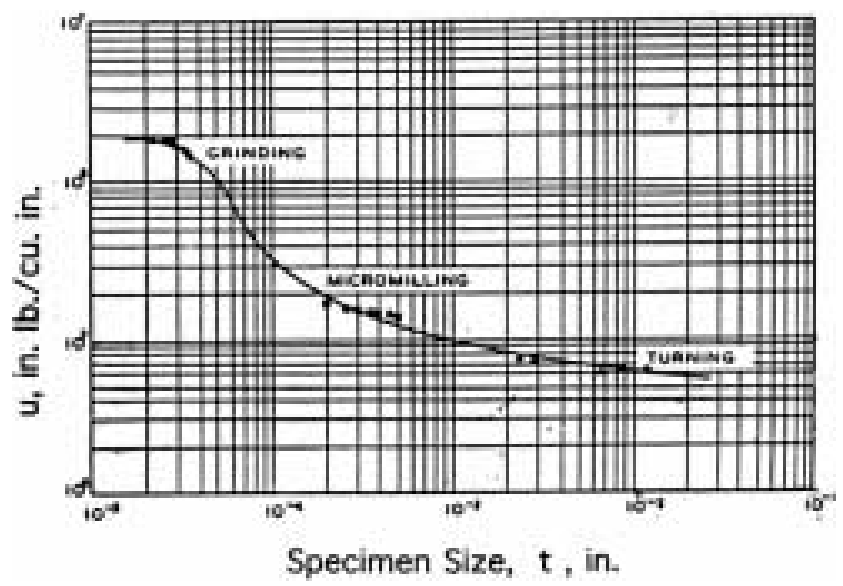

Figure 3. Variation of shear stress on shear plane when cutting SAE 1112 steel (after Backer et al 1952).

Horizontal and vertical forces were measured for a number of depths of cut $(t)$ when machining the same-sized surface as in grinding. The shear stress on the shear plane $(\tau)$ was estimated by a rather detailed method presented by Backer et al (1952).

Turning tests were performed upon a 2.25 in $(5.72 \mathrm{~cm})$ diameter SAE 1112 steel bar premachined in the form of a thin-walled tube having a wall thickness of 0.2 in $(5 \mathrm{~mm})$. A $0^{\circ}$ rake angle carbide tool was operated in a steady state two-dimensional orthogonal cutting mode as it machined the end of the tube. Values of shear stress on the shear plane $(\tau)$ versus undeformed chip thickness were determined for tests at a constant cutting speed and different values of axial infeed rate and for variable cutting speeds and a constant axial infeed rate. The grinding, micromilling and turning results are shown in figure 3.

A true stress-true strain tensile test was performed on a 0.505 in $(1.28 \mathrm{~cm})$ diameter by 2 in. $(5.08 \mathrm{~cm})$ gauge length specimen of the same SAE 1112 steel. The mean shear stress at fracture was $22,000 \mathrm{psi}(151.7 \mathrm{MPa})$. This value is not shown in figure 3 since it falls too far to the right.

In 1993 Dr. Norio Taniguchi was the American Society of Precision Engineers' distinguished lecturer in Seattle, WA. In his lecture discussing the art of nanotechnology (Taniguchi 1994), Dr. Tanigiuchi discusses the size effect in cutting and forming and presents his version of figure 3 in figure 4 which is more complete since it includes the tensile test.

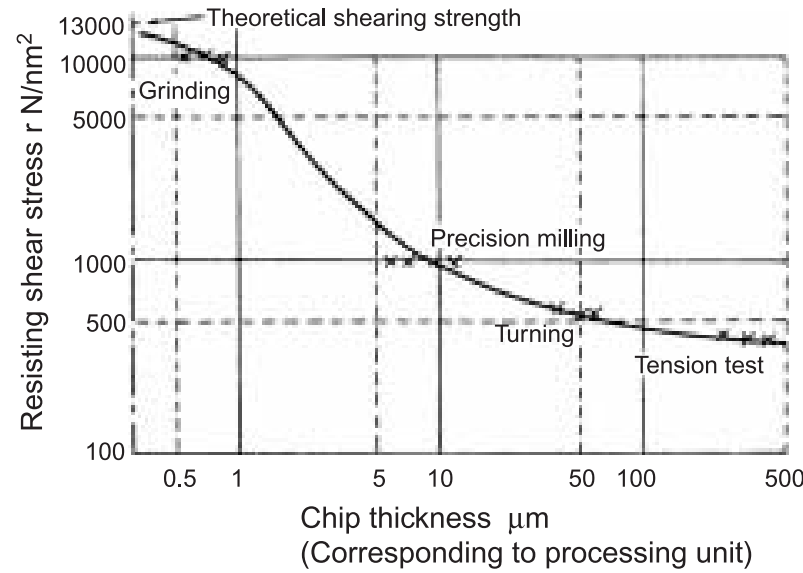

Figure 4. Relation between chip thickness and resisting shear stress for figure 3 as modified by Taniguchi (1994). 


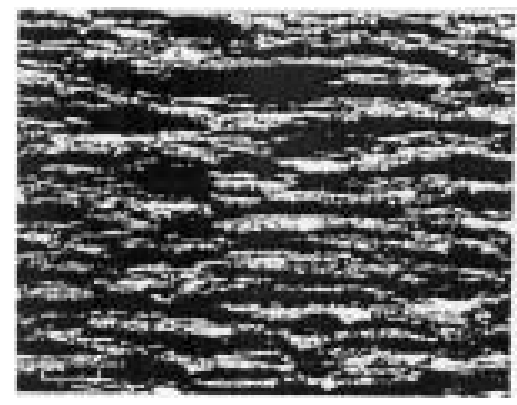

Figure 5. Back free surface of chip showing regions of discontinuous strain or microfracture.

Shaw (1952) discusses the origin of the size effect in metal cutting which is believed to be primarily due to short range inhomogeneities present in all commercial engineering metals. When the back of a metal cutting chip is examined at very high magnification by means of an electron microscope individual slip lines are evident as shown in figure 5. In deformation studies Heidenreich \& Shockley (1948) found that slip does not occur on all atomic planes but only on certain discrete planes. In experiments on deformed aluminum single crystals the minimum spacing of adjacent slip planes was found to be approximately 50 atomic spaces while the mean slip distance along the active slip planes was found to be about 500 atomic spaces as shown in figure 6 . These experiments further support the observation that metals are not homogeneous and suggest that the planes along which slip occurs are associated with inhomogeneities in the metal.

Strain is not uniformly distributed in many cases. For example, the size effect in a tensile test is usually observed only for specimens less than 0.1 in. $(2.5 \mathrm{~mm})$ in diameter. On the other hand, a size effect in a torsion test occurs for considerably larger samples due to the greater stress gradient present in a torsion test than in a tensile test. This effect and several other related ones are discussed in detail by Shaw (1952).

\section{Shear angle prediction}

There have been many notable attempts to derive an equation for the shear angle $(\phi)$ shown in figure 7 for steady state orthogonal cutting. Ernst \& Merchant (1941) presented the first quantitative analysis. Figure 7 shows the forces acting on a chip at the tool point where

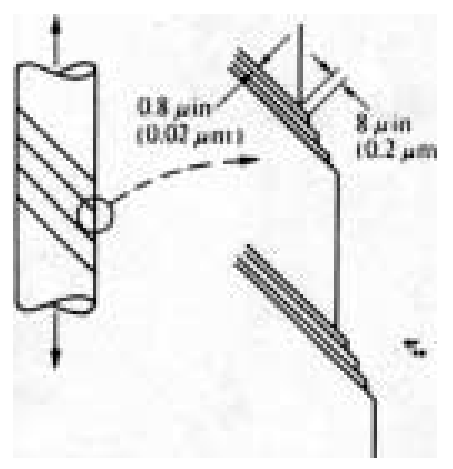

Figure 6. Spacing of adjacent slip planes for pure aluminium single crystal (after Heidenreich \& Shockley 1948). 


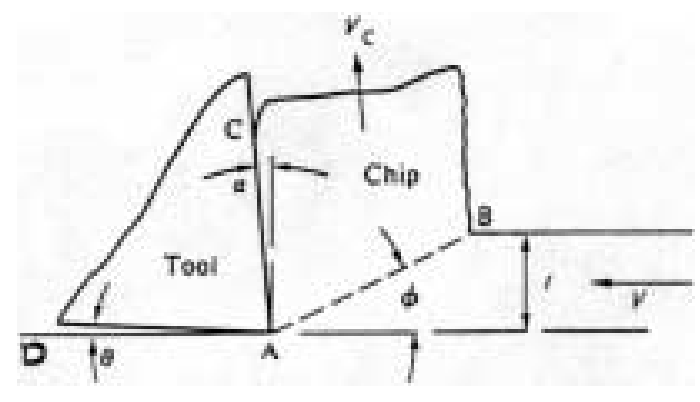

Figure 7. Nomenclature for two-dimensional steady state orthogonal cutting process.

$R$ is the resultant force on the tool face,

$R^{\prime}$ is the resultant force $\sim n$ the shear plane,

$N_{C}$ and $F_{C}$ are the components of $R$ normal to and parallel to the tool face,

$N_{S}$ and $F_{S}$ are the components of $R^{\prime}$ normal to and parallel to the cutting direction,

$F_{Q}$ and $F_{P}$ are the components of $R$ normal to and parallel to the cutting direction,

$\beta=\tan ^{-1} F_{C} / N_{C}$ (called the friction angle).

Assuming the shear stress on the shear plane $(\tau)$ to be uniformly distributed it is evident that

$$
\tau=F_{S} / A_{S}=\left(R^{\prime} \cos (\phi+\beta-\alpha) \sin \phi\right) / A,
$$

where $A_{S}$ and $A$ are the areas of the shear plane and that corresponding to the width of cut $(b)$, times the depth of cut $(t)$. Ernst \& Merchant (1941) reasoned that $\phi$ should be an angle such that $t$ would be a maximum and a relationship for $\phi$ was obtained by differentiating (1) with respect to $\phi$ and equating the resulting expression to zero. This led to:

$$
\phi=45-(\beta / 2)+(\alpha / 2) .
$$

However, it is to be noted that in differentiating, both $R^{\prime}$ and $\beta$ were considered independent of $\phi$.

Merchant (1945) presented a different derivation that also led to (2). This time an expression for the total power consumed in the cutting process was first written

$$
P=F_{P} V=(\tau A V)[\cos (\beta-\alpha) / \sin \phi \cos (\phi+\beta-\alpha)] .
$$

It was then reasoned that $\phi$ would be such that the total power would be a minimum. An expression identical to (2) was obtained when $P$ was differentiated with respect to $\phi$, this time considering $\tau$ and $\beta$ to be independent of $\phi$.

Piispanen (1937) had done this previously in a graphical way. However he immediately carried his line of reasoning one step further and assumed that the shear stress $\tau$ would be influenced directly by normal stress on the shear plane $\sigma$ as follows:

$$
\tau=r_{0}+K_{\sigma},
$$

where $K$ is a material constant. Piispanen then incorporated this into his graphical solution for the shear angle.

Upon finding (2) to be in poor agreement with experimental data Merchant also independently (without knowledge of Piispanen's work at the time) assumed the relationship given in (4), and proceeded to work this into his second analysis as follows: 


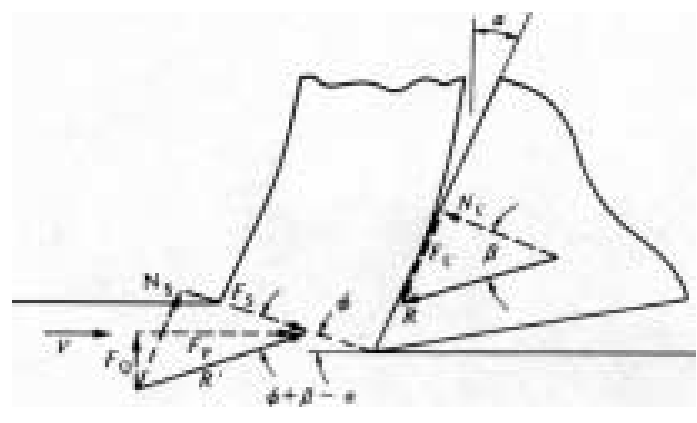

Figure 8. Cutting forces at the tool tip for the cutting operation in figure 7.

From figure 8 it may be seen that

$$
\sigma=\tau \tan (\phi+\beta-\alpha)
$$

or from (4)

$$
r_{0}=r+k_{\sigma} \tau \tan (\phi+\beta-\alpha)
$$

hence

$$
\tau=\frac{\tau_{0}}{1-K \tan (\phi+\beta-\alpha)}
$$

When this is substituted into (3) we have:

$$
P=\frac{\tau_{0} A V \cos (\beta-\alpha)}{[1-K \tan (\phi+\beta-\alpha)] \sin \phi \cos (\phi+\beta-\alpha)}
$$

Now, when $P$ is differentiated with respect to $\phi$ and equated to zero (with $\tau_{0}$ and $p$ considered independent of $\phi$ ) we obtain:

$$
\phi=\frac{\cot ^{-1}(K)}{2}-\frac{\beta}{2}+\frac{\alpha}{2}=\frac{C-\beta+\alpha}{2} .
$$

Merchant called the quantity $\cot ^{-1} K$ the machining 'constant' $C$. In figure 9 the quantity $C$ is seen to be the angle the assumed line relating $\tau$ and $\sigma$ makes with the $\tau$ axis.

Figure 10a shows the variation of shear stress on the shear plane versus compressive stress on the shear plane for a range of cutting conditions when SAE 9445 steel is turned with a carbide tool. The value of $C$ in (9) is seen to be $77^{\circ}$ to a good approximation. Figure 10b

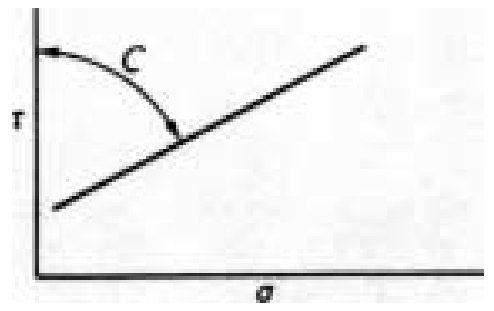

Figure 9. Relation between shear stress and normal stress on the shear plane assumed by Piispanen (1937) and by Merchant (1945) independently. 

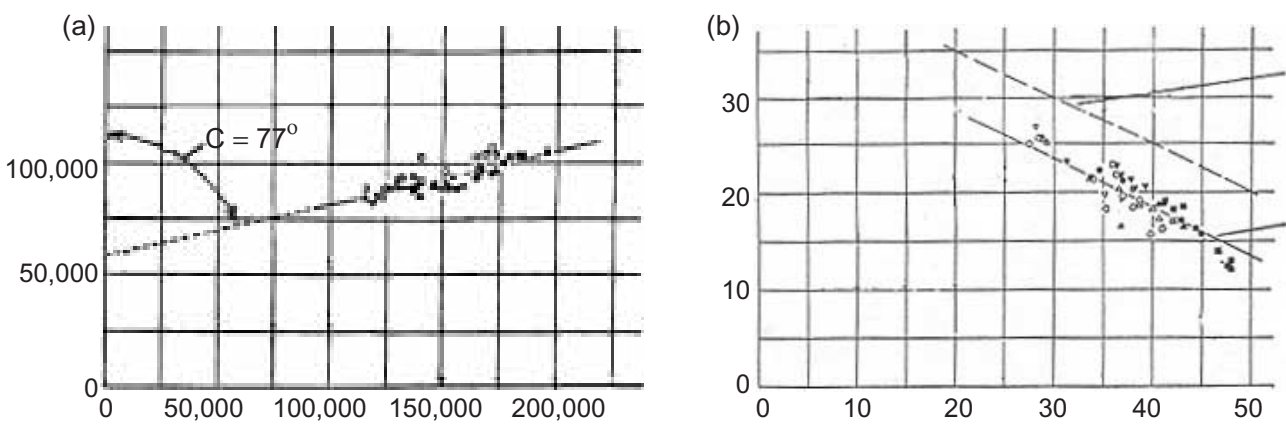

Figure 10. (a) Shear stress on shear plane versus compressive stress on shear plane (psi) for SAE 9445 steel machined with carbide tool. (b) Observed shear angle $\phi$ vs $\beta-\alpha$ (Merchant 1945b). Open points, $\alpha=+10^{\circ}$; solid points, $\alpha=-10^{\circ} ;{ }^{\circ} V=542 \mathrm{fpm}(165 \mathrm{mpm})$, variable $t ; \square t=0.0018 \mathrm{in} .(45.7 \mu \mathrm{m})$, variable $V ; \Delta t=0.0037$ in. $(94 \mu \mathrm{m})$, variable $V ; \nabla t=0.0062$ in. $(157 \mu \mathrm{m})$, variable $V$.

shows a comparison of the experimental data based on (2) and (9). Figure 11 shows similar results for SAE 4340 steel where the value of $C$ is seen to be $80^{\circ}$ to a good approximation.

Merchant (1950) has determined the values of $C$ given in Table 1 for materials of different chemistry and structure being turned under finishing conditions with different tool materials. From this table it is evident that $C$ is not a constant. Figures 10 and 11 illustrate how use of the Merchant empirical machining 'constant' $C$ that gives rise to (9) with values of $\phi$ in reasonably good agreement with experimentally measured values.

While it is well established that the rupture stress of both brittle and ductile materials is increased significantly by the presence of compressive stress (known as the Mohr Effect), it is generally believed that a similar relationship for flow stress does not hold.

Table 1. Values of $C$ in (9) for a variety of work and tool materials in finish turning without a cutting fluid.

\begin{tabular}{lcc}
\hline Work material & Tool material & C(deg) \\
\hline SAE 1035 Steel & HSS* & 70 \\
SAE 1035 Steel & Carbide & 73 \\
SAE 1035 Steel & Diamond & 86 \\
AISI 1022 (leaded) & HSS* & 77 \\
AISI 1022 (leaded) & Carbide & 75 \\
AISI 1113 (sul.) & HSS* & 76 \\
AISI 1113 (sul.) & Carbide & 75 \\
AISI 1019 (plain) & HSS* & 75 \\
AISI 1019 (plain) & Carbide & 79 \\
Aluminium & HSS* & 83 \\
Aluminium & Carbide & 84 \\
Aluminium & Diamond & 90 \\
Copper & HSS* & 49 \\
Copper & Carbide & 47 \\
Copper & Diamond & 64 \\
Brass & Diamond & 74 \\
\hline
\end{tabular}

${ }^{*} \mathrm{HSS}=$ high speed steel 

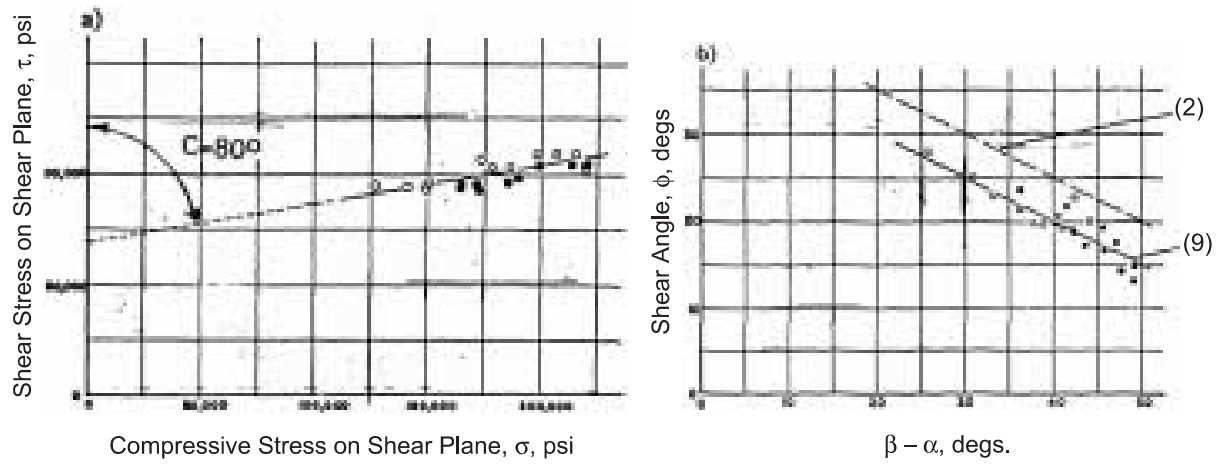

Figure 11. (a) Shear stress on shear plane versus compressive stress on shear plane for SAE 4340 steel machined with tungsten carbide tool. (b) Observed shear angle $\phi$ vs. $\beta-\alpha$ (after Merchant 1945b). Open points, $\alpha=+10^{\circ}$; solid points, $\alpha=-10^{\circ} ;{ }^{\circ} V=542 \mathrm{fpm}$ $(165 \mathrm{mpm})$, variable $t ; \square t=0.0018 \mathrm{in} .(45.1 \mu \mathrm{m})$, variable $v ; \Delta t=0.0031 \mathrm{in} .(94 \mu \mathrm{m})$, variable $v$; $\nabla \pm=0.0062$ in. $(159 \mu \mathrm{m})$, variable $v$.

However an explanation for this paradox with considerable supporting experimental data is presented below.

The fact that this discussion is limited to steady state chip formation rules out the possibility of periodic gross cracks being involved. However, the role of micro-cracks is a possibility consistent with steady state chip formation and the influence of compressive stress on the flow stress in shear. A discussion of the role micro-cracks can play in steady state chip formation is presented in the next section.

Hydrostatic stress plays no role in the plastic flow of metals if they have no porosity. Yielding then occurs when the von Mises criterion reaches a critical value. Merchant (1945) has indicated that Barrett (1943) found that for single crystal metals $\tau_{S}$ is independent of $\sigma_{S}$ when plastics such as celluloid are cut.

In general, if a small amount of compressibility is involved yielding will occur when the von Mises criterion reaches a certain value. However based on the results of figures 10 and 11 and Table 1 the role of compressive stress on shear stress on the shear plane in steady state metal cutting is substantial. The fact there is no outward sign of voids or porosity in steady state chip formation of a ductile metal during cutting and yet there is a substantial influence of normal stress on shear stress on the shear plane represents an interesting paradox. It is interesting to note that Piispanen (1937) had assumed that shear stress on the shear plane would increase with normal stress and had incorporated this into his graphical treatment. This was unknown to Merchant when writing his 1942 papers since a translation of Piispanen's paper from Finnish into English became available only between the time Merchant's paper was written and when it was published. It is not uncommon that important discoveries are made quite independently at different locations at about the same time. A case in point is discovery of the dislocation quite independently by Orowan (1934), Polanyi (1934) and Taylor (1934). Apparently there is a right time for important discoveries to be made!

\section{Plastic behaviour at large strain}

There has been remarkably little work done in the region of large plastic strains. Nobel Laureate Bridgman (1952), using the hollow tubular notched specimen shown in figure 12, 


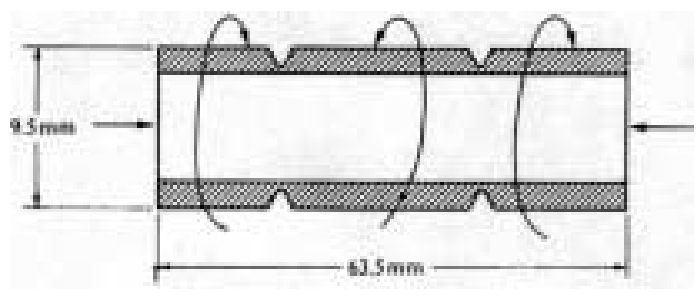

Figure 12. Bridgman (1952) specimen for combined axial load and torsion.

performed tests under combined axial compression and torsion. The specimen was loaded axially in compression as the centre section was rotated relative to the ends. Strain was concentrated in the reduced sections and it was possible to crudely estimate and plot shear stress vs. shear strain with different amounts of compressive stress on the shear plane. From these tests, Bridgman concluded that the flow curve for a given material is the same for all values of compressive stress on the shear plane, a result consistent with other materials tests involving much lower plastic strains. However, the strain at gross fracture is found to be strongly influenced by compressive stress. A number of related results are considered in the following subsections.

\subsection{Langford and Cohen}

Langford \& Cohen (1969) were interested in the behaviour of dislocations at very large plastic strains and whether there is saturation relative to the strain hardening effect with strain or whether strain hardening continues to occur with strain to the point of fracture.

Their experimental approach was an interesting and fortunate one. They performed wire drawing on iron specimens using a large number of progressively smaller dies with remarkably low semi-die angle $\left(1.5^{\circ}\right)$ and a relatively low $(10 \%)$ reduction in area per die pass. After each die pass, a specimen was tested in uniaxial tension and a true stress-strain curve obtained. The drawing and tensile tests were performed at room temperature and low speeds to avoid heating and specimens were stored in liquid nitrogen between tests to avoid strain-aging effects. All tensile results were then plotted in a single diagram, the strain used being that introduced in drawing ( 0.13 per die pass) plus the plastic strain in the tensile test. The result is shown in figure 13a. The general overlap of the tensile stress-strain curves gives an overall strain hardening envelope which indicates that the wire drawing and tensile deformations are approximately equivalent relative to strain hardening. Figure $13 \mathrm{~b}$ shows similar results on the same iron wire tested in uniaxial compression following drawing.

Figure 13c shows somewhat similar results obtained earlier by Blazynski \& Cole (1960) for AISI 1012 steel carried to much lower values of total strain. Blazynski \& Cole (1960) were interested in strain hardening in tube drawing and tube sinking. Drawn tubes were sectioned as shown in figure $13 \mathrm{~d}$ and tested in plane strain compression as shown in figure $13 \mathrm{e}$. Figure $13 \mathrm{c}$ shows the flow stress in compression plotted against the total strain.

The curves with bent tops in figure $13 \mathrm{c}$ were obtained using graphite grease as a lubricant in the plane strain tests while the data points were obtained in similar tests using a more effective molybdenum disulphide lubricant. The smooth curve drawn through the molybdenum disulphide data points constitutes the flow curve essentially in the absence of friction.

Up to a strain of about 1 (figures $13 \mathrm{a}, \mathrm{b}$ and $\mathrm{c}$ ) the usual strain-hardening curve is obtained that is in good agreement with the generally accepted equation,

$$
\sigma=\sigma_{1} \varepsilon^{n} .
$$



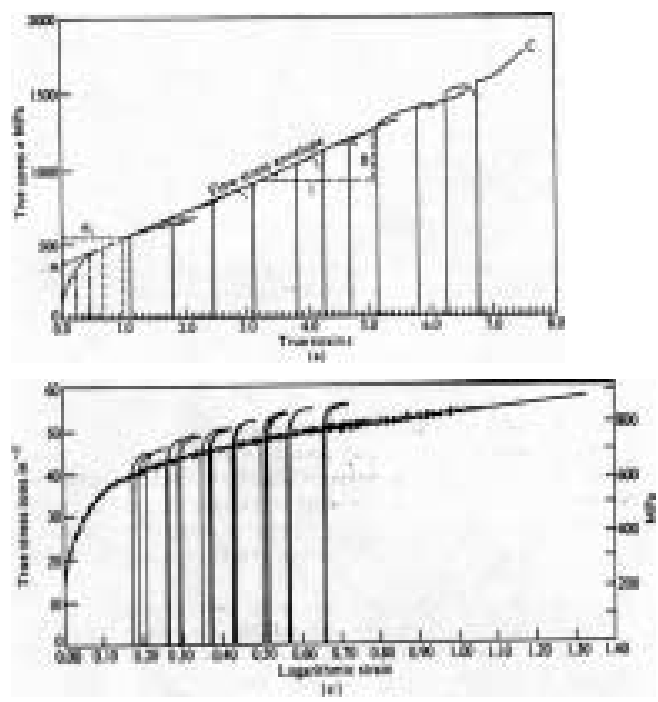
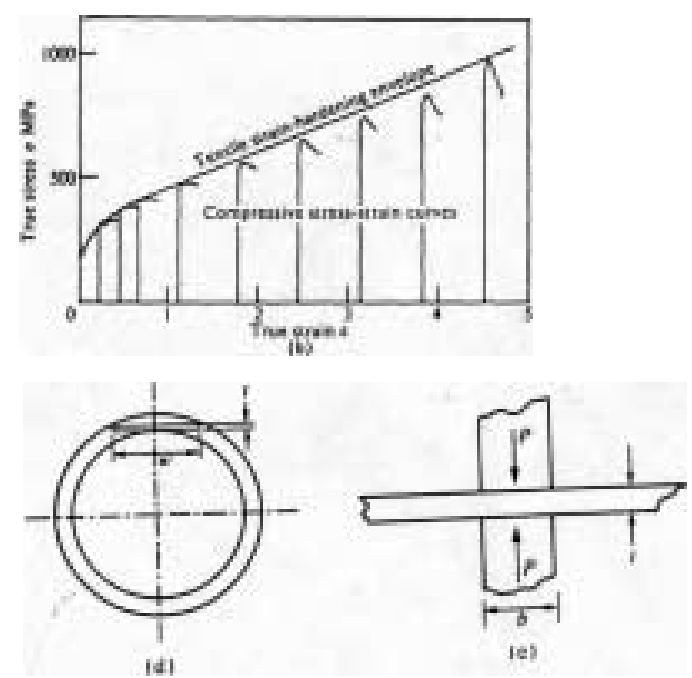

(e)

Figure 13. (a) Effect of wire drawing strain on uniaxial stress-strain curve (corrected for necking) of furnace cooled iron ( 0.007 w/o C) drawn and tested at room temperature (after Langford \& Cohen 1969). (b) Uniaxial compression test data following wire drawing of same materials (tested in tension following drawing) shown in (a). (c) Plane strain compression tests following tube drawing of AISI 1012 steel. Curves with hooks at top are with graphite lubricant while data points are for a more effective molybdenum disulphide lubricant (after Blazynski \& Cole 1960). (d) How plane strain specimen was removed from the drawn tube by Blazynski \& Cole (1960). Tube diameter was approximately $55 \mathrm{~mm}$, wall thickness $4.8 \mathrm{~mm}$. (e) Plane strain compression test used by Blazynski \& Cole (1960) following tube drawing. $t=0.045 \mathrm{in} .(1.14 \mathrm{~mm}) ; b=0.115 \mathrm{in} .(2.92 \mathrm{~mm}), w$ (perpendicular to paper $=0.690 \mathrm{in}$. $(17.53 \mathrm{~mm})$.

However, beyond a strain of 1 , the curve was linear corresponding to the equation

$$
\sigma=A+B \varepsilon,(\varepsilon<1)
$$

where $A$ and $B$ are constants. It may be shown that

$$
\begin{aligned}
& A=(1-n) \sigma_{1}, \\
& B=n \sigma_{1},
\end{aligned}
$$

in order that the curves of (10) and (11) have the same slope and and ordinate at $\varepsilon=1$.

While (10) is well-known and widely applied there is relatively little data in the literature for plastic strains greater than 1 and hence (11) is relatively unknown.

From transmission electron micrographs of deformed specimens, Langford \& Cohen (1952) found that cell walls representing concentrations of dislocations began to form at strains below 0.2 and became ribbon-shaped with decreasing mean linear intercept cell size as the strain progressed. Dynamic recovery and cell wall migration resulted in only about $7 \%$ of the original cells remaining after a strain of 6 . The flow stress of the cold-worked wires was found to vary linearly with the reciprocal of the mean transverse cell size.

Data that make one question the wisdom of extrapolating test data from ordinary materials to the larger strain regime of metal cutting are given in figure 14 . These are data for the same material cut under the same conditions except for rake angle $(\alpha)$. The shear stress on the shear plane is obviously not constant but appears to decrease with increase in shear strain (negative strain hardening). 


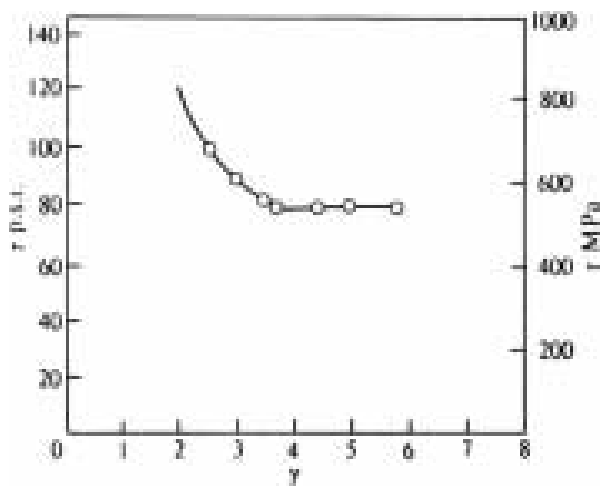

Figure 14. Values of shear stress on shear plane versus shear strain in chip when cutting the same material with tools of different rake angle (after Shaw 1954).

\subsection{Walker \& Shaw (1967)}

Toward the end of the sixties it was decided to conduct an acoustical emission study of concentrated shear at Carnegie-Mellon University. The initial acoustical studies were on specimens of the Bridgman type but, fortunately, lower levels of axial compressive stress than Bridgman had used, were employed in order to more closely simulate the concentrated shear process of metal cutting. The apparatus used, which was capable of measuring stresses and strains as well as acoustical signals arising from plastic flow, is described in the dissertation of Walker (1967). Two important results were obtained.

(1) A region of rather intense acoustical activity occurs at the yield point followed by a quieter region until a shear strain of about 1.5 is reached. At this point there is a rather abrupt increase in acoustical activity that continues to the strain at fracture which is appreciably greater than $1 \cdot 5$;

(2) The shear stress appears to reach a maximum at strain corresponding to the beginning of the second acoustical activity $(\gamma=1.5)$

The presence of the notches in the Bridgman specimen (figure 12) made interpretation of stress-strain results somewhat uncertain. Therefore, a new specimen was designed (figure 15) which substitutes simple shear for torsion with normal stress on the shear plane. By empirically adjusting distance $\Delta x$ (figure 15) to a value of $0.25 \mathrm{~mm}$ it is possible to confine all the plastic shear strain to the reduced area, thus making it possible to readily determine the shear strain $(\gamma=\Delta y / \Delta x)$. When the width of the minimum section is greater or lesser than $0.25 \mathrm{~mm}$, the extent of plastic strain observed in a transverse micrograph at the minimum section either does not extend completely across the $0.25 \mathrm{~mm}$ dimension or is beyond this width.

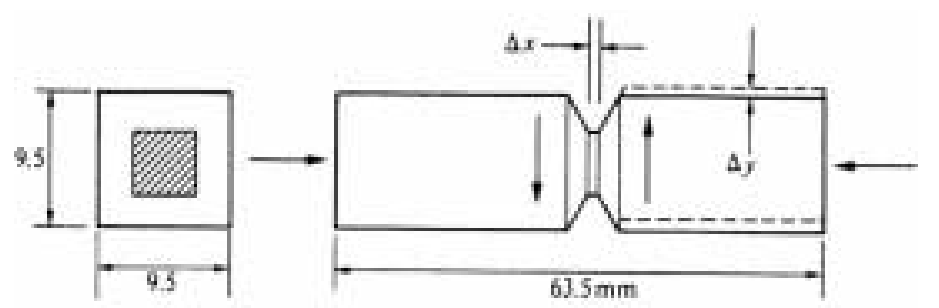

Figure 15. Plane strain simple shear-compression specimen of Walker \& Shaw (1969). 


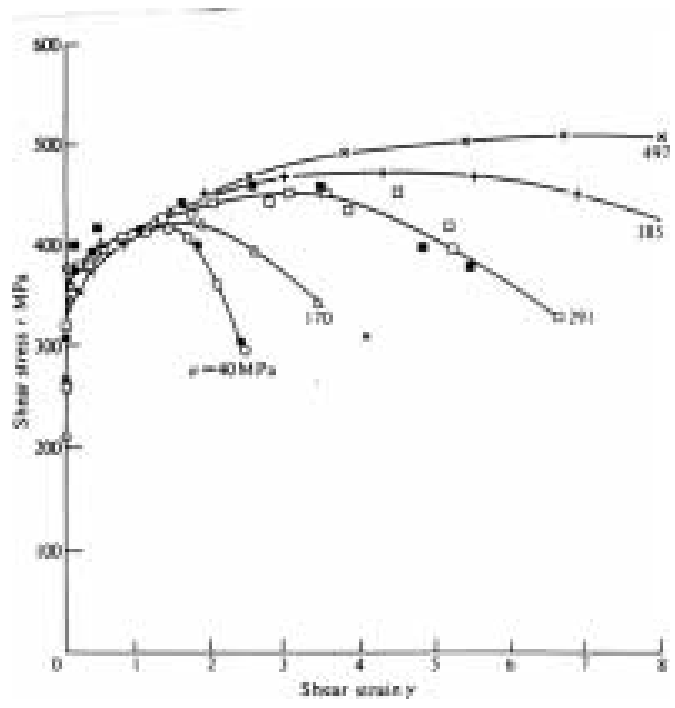

Figure 16. Shear stress-shear strain results for resulphurised low carbon steel for specimen of figure 15 (after Walker \& Shaw 1969).

A representative set of curves is shown in figure 16 for resulphurised low carbon steel. Similar results are obtained for nonresulphurised steels and other ductile metals. There is little difference in the curves for different values of normal stress on the shear plane $(\sigma)$ to a shear strain of about 1.5 .

This is in agreement with Bridgman (1952). However, beyond this strain, the curves differ substantially with compressive stress on the shear plane. At large strains $(\tau)$ is found to decrease with increase in $(\gamma)$, a result that does not agree with Bridgman (1952).

When the results of figure 13a and 16 are compared they are seen to be very different. In the case of figure 13a, strain hardening is positive to normal strains as high as 7 . In the case of figure 16 strain hardening becomes negative above a particular shear strain that increases with normal stress on the shear plane.

From figure 16 it is seen that for a low value of normal stress on the shear plane of $40 \mathrm{MPa}$ strain hardening appears to be negative at a shear strain of about $1 \cdot 5$; that is, when the normal stress on the shear plane is about $10 \%$ of the maximum shear stress reached, negative strain hardening sets in at a shear strain of about 1.5. On the other hand, strain hardening remains positive to a normal strain of about 8 when the normal stress on the shear plane is about equal to the maximum shear stress (note curve for $\sigma=497 \mathrm{MPa}$ in figure 16).

\subsection{Usui et al (1961)}

Usui et al (1961) describe an experiment designed to determine why $\mathrm{CCI}_{4}$ is such an effective cutting fluid at low cutting speeds. Since this also has a bearing on the role of microcracks in large strain deformation, it is considered here.

A piece of copper was prepared as shown in figure 17. The piece that extends upward and appears to be a chip is not a chip but a piece of undeformed material left there when the specimen was prepared. A vertical flat tool was then placed precisely opposite the free surface as shown in figure 17 and fed horizontally. Horizontal $F_{P}$ and vertical $F_{Q}$ forces were recorded as the shear test proceeded. It was expected that the vertical piece would fall free from the lower material after the vertical region had been displaced a small percentage of its length. However, it went well beyond the original extent of the shear plane and was still 


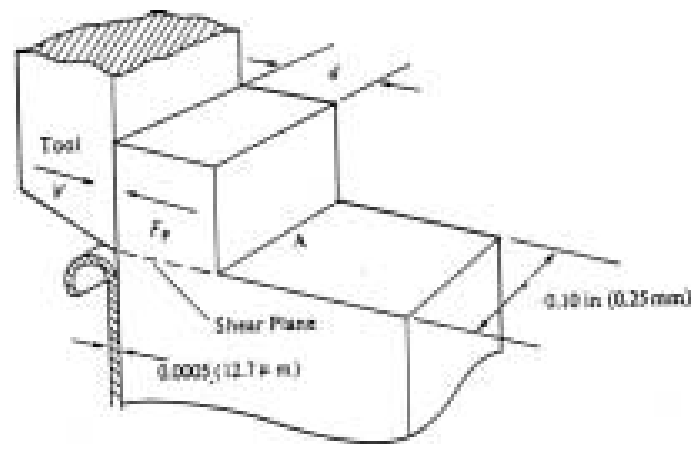

Figure 17. Special shear test arrangement (after Usui et al 1961).

firmly attached to the base. This represents a huge shear strain since the shear deformation is confined to a narrow band. When a single drop of $\mathrm{CCI}_{4}$ was placed at $A$ in figure 17 before conducting the shear test the protrusion could be moved only a fraction of the displacement in air before gross fracture occurred on the shear plane. Figure 18 shows photo-micrographs of tests without and with $\mathrm{CCI}_{4}$. It is apparent that $\mathrm{CCI}_{4}$ is much more effective than air in preventing microcracks from rewelding.

\subsection{Saw tooth chip formation in hard turning}

Saw tooth chip formation for hard steel discussed by Vyas and Shaw (1999) is another example of the role microcracks play. In this case, gross cracks periodically form at the free surface and run down along the shear plane until sufficient compressive stress is encountered to cause the gross crack to change to a collection of isolated microcracks.

\subsection{Fluid-like flow in metal cutting chip formation}

At the General Assembly of the International Institution for Production Engineering Research (CIRP) in 1952, an interesting paper was presented by Eugene (1952). Figure 19 shows the
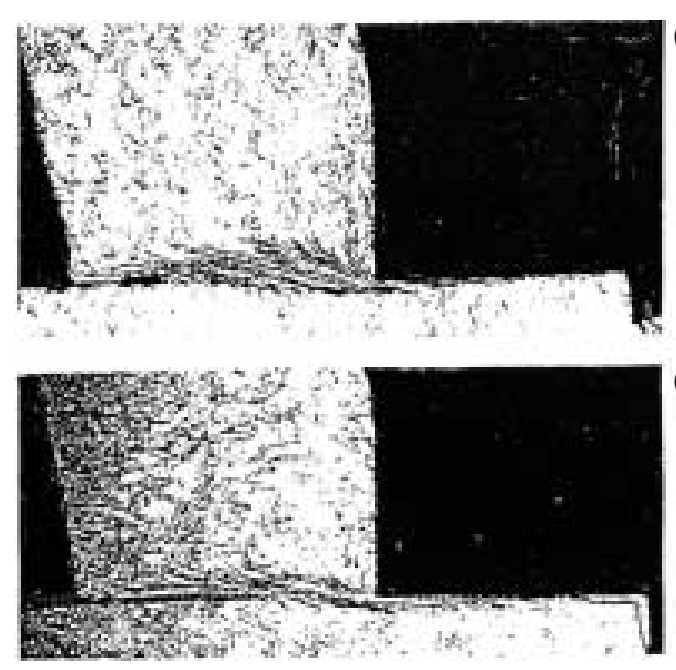

(a)

(b)
Figure 18. Photomicrographs of specimens that have been sheared a distance approximately equal to the shear plane length: (a) In air, (b) with a drop of $\mathrm{CCL}_{4}$ applied at point $A$ in figure 17 . 


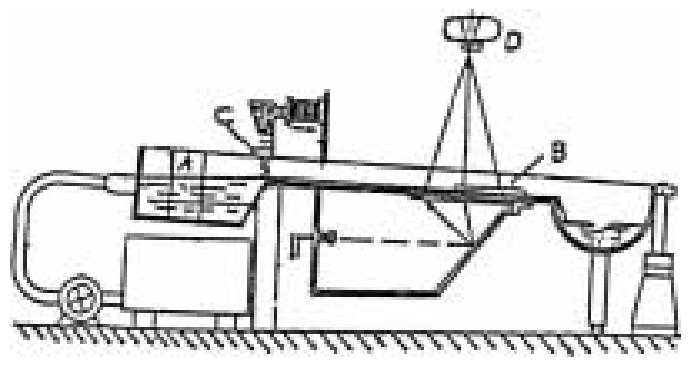

Figure 19. Apparatus used by Eugene (1952) to photograph flow paths past tools having different rake angles.

apparatus he used. Water is pumped into a baffle chamber $A$ that removed eddies and then causes flow under gravity past a simulated tool at $B$. Powdered bakelite is introduced at $C$ to make the streamlines visible as the fluid flows past the tool. The photographs taken by the camera at $D$ are remarkably similar to quick stop photomicrographs of actual chips. It was thought by this author at the time that any similarity between fluid flow and plastic flow of a solid was not to be expected. That was long before it was clear that the only logical explanation for the results of Bridgman and Merchant involve microfracture (Shaw 1980).

At the General Assembly of CIRP 47 years later, a paper was presented that again suggests that metal cutting might be modelled by a fluid (Kwon et al 1999). However, this paper is concerned with ultraprecision machining (depths of cut $<4 \mu \mathrm{m}$ ) and potential flow analysis is employed instead of the experimental approach taken by Eugene.

It is interesting to note that chemists relate the flow of liquids to the migration of vacancies (voids) just as physicists relate ordinary plastic flow of solid metals to the migration of dislocations. Henry Eyring and coworkers (Eyring et al 1958; Eyring \& Ree 1961; Eyring \& Jhon 1969) have studied the marked changes in volume, entropy and fluidity that occur when a solid melts. For example, a $12 \%$ increase in volume accompanies melting of argon, suggesting the removal of every eighth molecule as a vacancy upon melting. This is consistent with X-ray diffraction of liquid argon that shows good short range order but poor long range order. The relative ease of diffusion of these vacancies accounts for the increased fluidity that accompanies melting. A random distribution of vacancies is also consistent with the increase in entropy observed on melting. Eyring's theory of fluid flow was initially termed the "hole theory of fluid flow" but later "the significant structure theory" which is the title of the Eyring-Jhon book (1969).

According to this theory, the vacancies in a liquid move through a sea of molecules. Eyring's theory of liquid flow is mentioned here since it explains why the flow of a liquid approximates the flow of metal past a tool in chip formation. In this case, microcracks (voids) move through a sea of crystalline solid.

\subsection{Kececioglu's contributions}

Kececioglu $(1958,1960)$ has presented several papers that illustrate the difficulty of predicting the mean shear stress on the shear plane in steady state chip formation. The last of these builds on the others and is most important relative to the modelling being discussed here. Based on a large number of dry-cutting experiments on AISI 1015 steel tubing having a hardness of 118 Brinell and using a steel cutting grade of carbide, Kececioglu (1960) concluded that the mean shear stress on the shear plane depends upon the following variables:

- mean normal stress on the shear plane $\left(\sigma_{S}\right)$

- shear volume of the shear zone $(e)$ 
- mean strain rate in the shear plane $(\dot{\gamma})$

- mean temperature of the shear plane $\left(\theta_{S}\right)$

- degree of strain hardening in the work before cutting

If this were not complicated enough, it is clear from Kececioglu's experimental results that these items do not act independently (i.e. the influence of a high normal stress on the shear plane on shear stress on the shear plane depends upon the combination of other variables pertaining). This is an important result since it means that in general it is not possible to extrapolate material test values of shear stress to vastly different cutting conditions.

It is interesting to note that Kececioglu $(1958,1960)$ suggests that the specific energy should be related to the shear plane area $(e)$ instead of $(t)$ as in most treatments of metal-cutting mechanics. The shear plane area is

$$
e=b(t / \sin \phi) \Delta y\left(\mathrm{in}^{3} \text {. or } \mathrm{mm}^{3}\right) \text {. }
$$

This appears to be a useful suggestion. In both cases the inverse relation between $u$ and $t$ or $e$ is due to the greater chance of encountering a stress-reducing defect as $t$ or $e$ increases. The use of $e$ instead of $t$ is a more general although more complex way of expressing the "size effect". The range of values covered by Kececioglu in his orthogonal experiments on AISI 1015 steel were as follows:

- rake angle: $-10^{\circ}$ to $+37^{\circ}$

- undeformed chip thickness $(t): 0.004$ to 0.012 in $(0.2$ to $0.3 \mathrm{~mm})$

- cutting speed $(V): 126$ to $746 \mathrm{fpm}(38.4$ to $227.4 \mathrm{~m} / \mathrm{min})$

- inclination angle $(i): 0$ to $35^{\circ}$

This resulted in the following wide range of dependent variables:

- width of shear zone: 0.002 to 0.007 in $(0.10$ to $0.18 \mathrm{~mm})$

- rate of strain: 20000 to $40000 \mathrm{~s}^{-1}$

- mean shear stress on shear plane $\left(\tau_{S}\right) 62,000$ to $84,000 \mathrm{psi}(427.5$ to $579.2 \mathrm{MPa})$

- mean normal stress on shear plane: 1000 to 12000 psi (6.9 to $83 \mathrm{MPa})$

- mean shear plane temperature: 410 to $840 \mathrm{~F}\left(210\right.$ to $\left.449^{\circ} \mathrm{C}\right)$

Figure 20a shows considerable scatter of the variation of shear stress with normal stress due to the fact that the effect of changing one independent variable on shear stress depends upon a combination of the other variables as well. Figure $20 \mathrm{~b}$ shows the variation of the mean value of shear stress for a group of points in figure 20a in the vicinity of the value of normal stress plotted in figure 20b. This indicates, on the average, that shear stress increases with increase in normal stress. This is consistent with the view that under the very high strains involved in metal cutting (a mean value of about 3.5 in Kececioglu's experiments) localised microcracks are likely to form on the shear plane and an increase in normal stress should decrease their number and give rise to an increase in shear stress on the shear plane.

Figures 21a and $\mathrm{b}$ show similar results for the mean shear stress on the shear plane versus the volume of the shear zone $(e)$. This shows clearly that in general a decrease in the shear zone volume causes an increase in the shear stress on the shear plane, particularly for shear volumes below $10^{-5} \mathrm{in}^{3}$.

Figure 22 shows results for the mean shear stress on the shear plane versus the shear strain for the tests of figures 20 and 21 . This yields the unusual result that the shear stress decreases with increase in strain which is not consistent with ordinary materials test results that involve strain hardening. The reason for this paradox is due to the fact that in addition to strain several other variables are involved and the net effect is a decrease in shear stress with shear strain. 
(a)

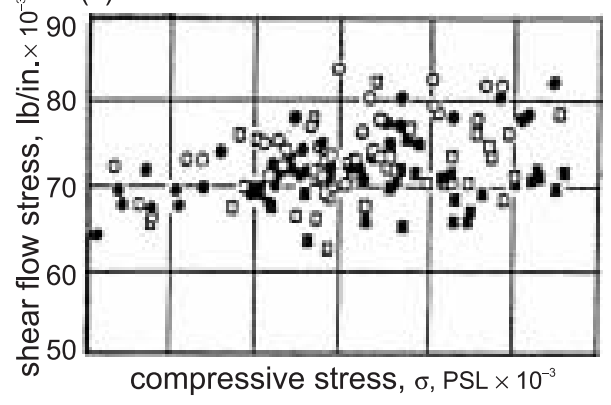

(b)

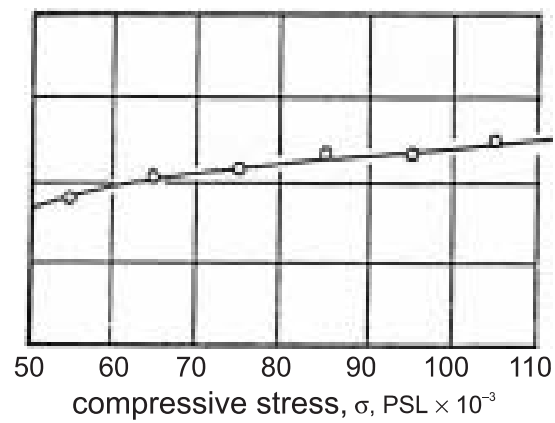

Figure 20. Variation of shear stress on the shear plane with normal stress on the shear plane, (a) for 43 tests involving a wide range of rake angle, cutting speed, undeformed chip thickness, and inclination angle, (b) mean values of shear stress on the shear plane for values of normal stress indicated (after Kececioglu 1960).

\subsection{Zhang \& Bagchi (1994)}

Zhang \& Bagchi (1994) have presented valuable analysis of the chip separation problem in FEM in terms of microfracture mechanics. This is based on the fact that ductile metals fail in three steps: nucleation, growth, and coalescence of microvoids that initiate at points of stress concentration (Anderson 1991). Figure 23 illustrates how the three steps lead to gross fracture in shear. Figure 24 shows a random distribution of defects (points of stress concentration) in a ductile metal chip. As the work material approaches a stationary tool, defects along the $x$ axis in the cutting direction are subjected to increasing stress. This leads to nucleation of a void at $A$ in figure $24 \mathrm{a}$, growth as the void moves to $B$ in figure $24 \mathrm{~b}$ and coalescence with the tool as shown in figure $24 \mathrm{c}$.

Zhang \& Bagchi (1994) suggest that voids approaching the tool tip grow but do not coalesce before reaching the tool tip. This is important since coalescence would lead to a gross crack extending in front of the tool tip and this is never observed experimentally even

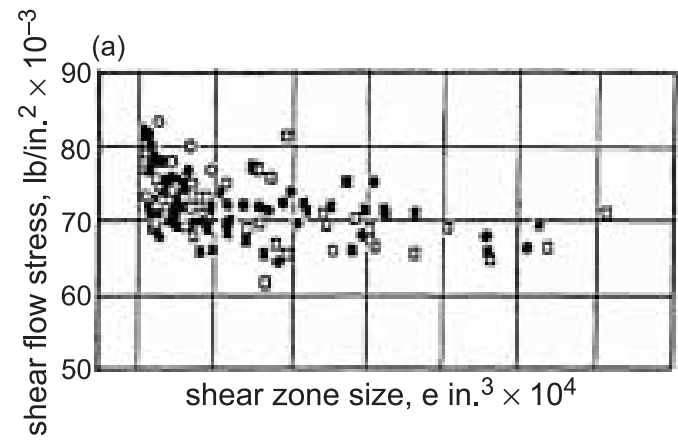

(b)

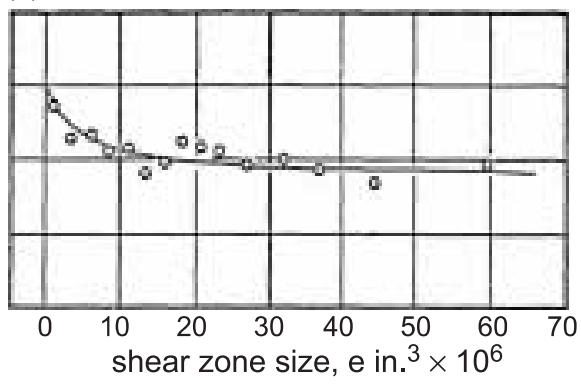

Figure 21. Variation of shear stress on shear plane with shear zone volume: (a) for 43 tests involving a wide range of rake angle, cutting speed, undeformed chip thickness, and inclination angle, (b) mean values of shear stress on the shear plane for values of shear volume close to the values of $e$ indicated (after Kececioglu 1960). 
?

(a)

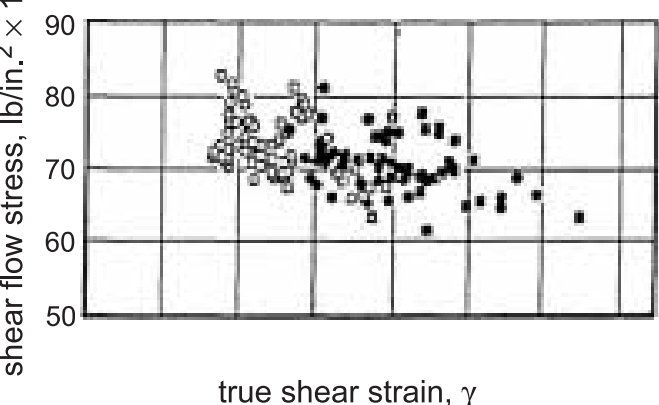

(b)

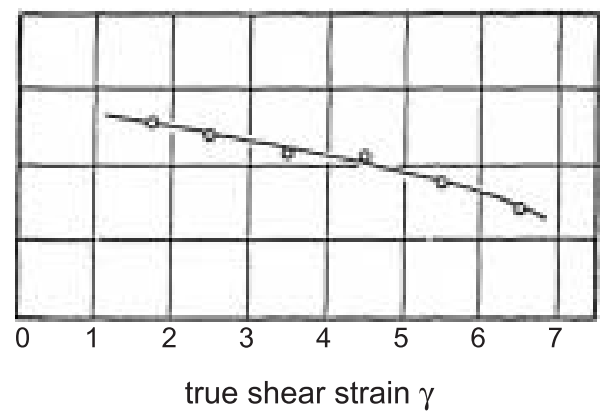

Figure 22. Variation of shear stress on the shear plane with shear strain on a shear plane. (a) For 43 tests involving a wide range of rake angle, cutting speed, undeformed chip thickness, and inclination angle, (b) mean values of shear stress on the shear plane for values of shear strain $(\gamma)$ close to the values of shear strain $(\gamma)$ indicated. (after Kececioglu 1960).

when hard materials are machined. Zhang \& Bagchi (1994) have applied the continuum model for void nucleation of Argon et al (1975) and that of Rice \& Tracey (1969) for void growth. It is assumed that separation occurs when the leading void reaches its maximum size.

The theory presented by Zhang \& Bagchi (1994) based on the presence of points of stress concentration, formation of microvoids, void growth and void coalescence within the tool tip offers a very reasonable explanation for the flow separation problem involved when FEM is applied to metal cutting chip formation.

Figure 25 shows a diagram equivalent to figure 24 including action along the shear plane. Here the material in the shear plane is subjected to very large strains and any points of stress concentration should be expected to give rise to microcracks instead of micovoids. In the

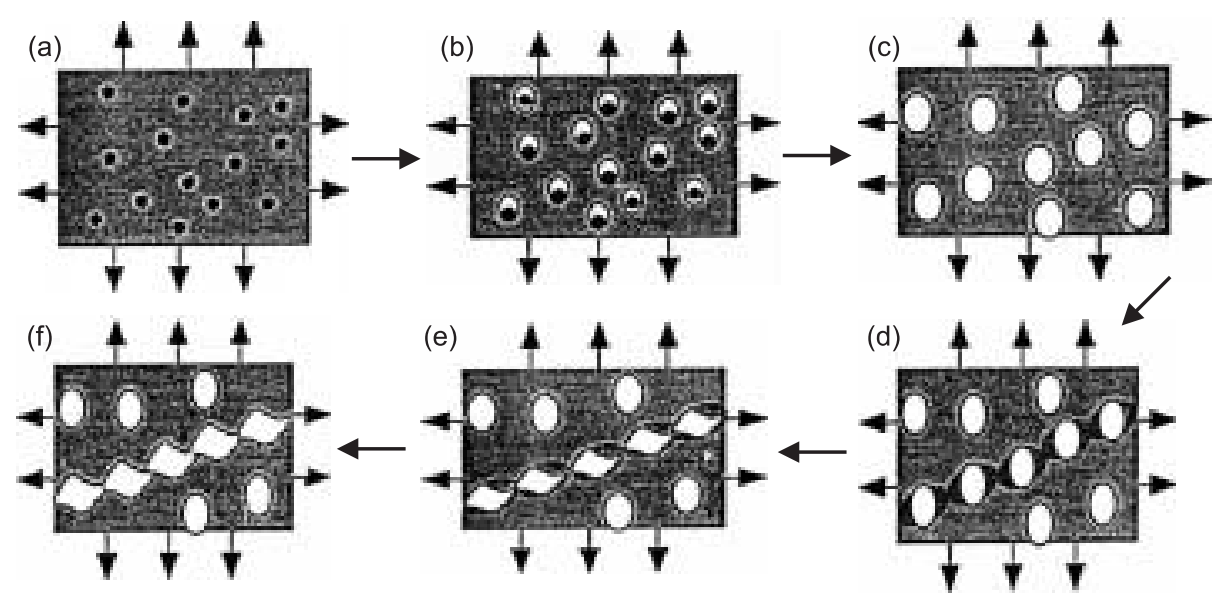

Figure 23. Void nucleation, growth, and coalescence in ductile metals (after Anderson 1991). (a) Inclusions in a ductile matrix, (b) void nucleation, (c) void growth, (d) strain localization between void, (e) necking between voids, (f) void coalescence and fracture. 

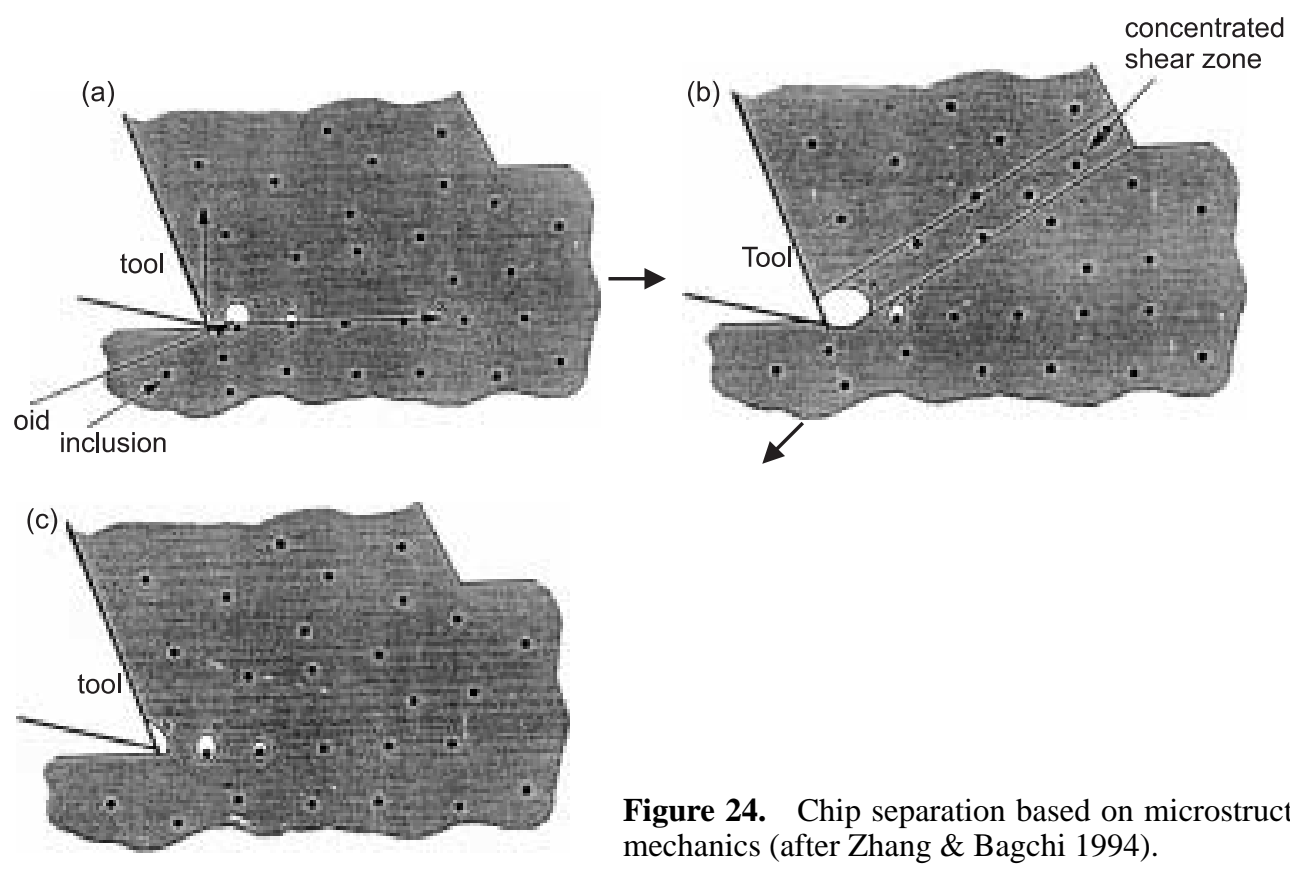

Figure 24. Chip separation based on microstructure mechanics (after Zhang \& Bagchi 1994).

presence of relatively high normal stress on the shear plane and the absence of a contaminating film such as $\mathrm{CCI}_{4}$ vapour, these microcracks reweld after moving a relatively short distance. Also, due to the very high strains associated with the shear plane very much higher density of microcracks is involved than the density of microvoids involved in the undeformed work material of figure 24 .

Based on the discussion presented, it is concluded that microcracks usually play an important role in steady state metal cutting chip formation because of the unusually large strains involved. Also, there is considerable experimental evidence that normal stress on the shear plane has a substantial influence on shear stress on the shear plane. Both of these rule out use of the von Mises criterion in metal cutting modelling except as a very rough approximation.

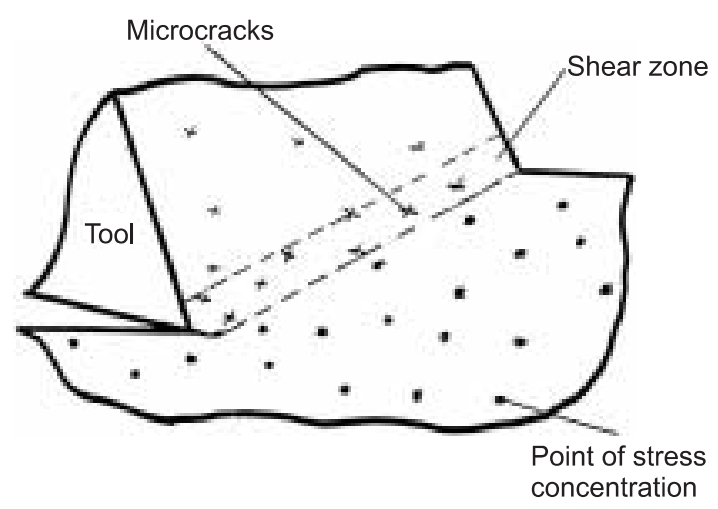

Figure 25. Chip formation involving transport of microcracks across the shear plane by formation, displacement and rewelding. 


\section{New mechanism of large strain plastic flow}

It is seen that whereas the Bridgman, Langford \& Cohen results are in agreement, these results are completely different from those of Walker \& Shaw. A proposed mechanism of large strain plastic flow (Shaw 1980) suggests that at moderate values of normal stress on the shear plane, discontinuous microcracks begin to appear in a plane of concentrated shear at a shear strain of about 1.5. As strain proceeds beyond this point the first microcracks are sheared shut as new ones take their place. The sound area on the shear plane gradually decreases until it becomes insufficient to resist the shear load without gross facture. What seems to be negative strain-hardening in figure 16 is due to what might be described as 'internal necking' (i.e. a gradual decrease in sound internal area with load just as the area in the neck of a tensile specimen decreases with load to give the appearance of negative strain-hardening in an engineering stress-strain curve).

The reason such 'negative strain hardening' was not observed by Bridgman or Langford \& Cohen appears to be due to the normal compressive stress on the shear plane in their experiments being high enough to prevent the formation of microcracks. The choice of a die angle (only $1.50^{\circ}$ half angle) and a reduction per pass $(0.22)$ in the Langford \& Cohen drawing experiments provides essentially homogeneous compressive strain in the deformation zone and under such conditions, one would not expect microcracks to develop. There is considerable indirect evidence to support the formation of microcracks in metal cutting. While it has been reported (Komanduri \& Brown 1967) that microcracks have been observed on the shear plane of quick-stop chip roots, one should not expect to find many. Such cracks will be of the thin hairline variety and most of them would be expected to reweld as the specimen is suddenly unloaded.

The new theory of plastic flow discussed here is an add-on to dislocation theory. As long as microcracks do not occur in appreciable number, a material may be deformed to very large strains with a continuous increase in dislocation density and strain hardening. This is consistent with the experimental results of Bridgman and Langford \& Cohen. However, at a particular value of shear strain, depending upon the ductility of the material and the normal stress on the shear plane, microcracks begin to form. If the normal stress on the shear plane is tensile, these cracks spread rapidly over the shear surface leading to gross fracture. If, however, a moderate compressive stress (for example $1 / 2$ the shear flow stress for a ductile metal) is present on the shear plane, the new mechanism pertains. When this occurs there is an extended stressstrain region exhibiting decrease in flow stress with strain as the ratio of real to apparent area on the shear plane $\left(A_{R} / A\right)$ decreases from one to the critical value at which gross fracture occurs.

It is suggested that in metal cutting the shear stress on the shear plane is not, in general, independent of normal stress on the shear plane. The part of the shear plane that involves microcracks should show an increase in shear stress with normal stress as Piispanen and Merchant suggested. However, the part that does not involve microcracks should have a shear stress that is independent of normal stress in keeping with the experimental results of Bridgman and Langord \& Cohen.

It should not be inferred that the foregoing discussion has brought us any nearer to the solution of the shear angle problem. It should, however, serve to further explain why it is unlikely that a simple solution to this persistent problem is apt to be found. 


\section{Inhomogeneous strain}

When metallic single crystals are plastically deformed as previously stated, it is found that slip does not occur uniformly on every atomic plane but that the active slip planes are relatively far apart (figure 6). It is further found that polycrystalline metals also strain blockwise rather than uniformly. Crystal imperfections are responsible for this inhomogeneous behaviour.

When the volume of material deformed at one time is relatively large, there is a uniform density of imperfections and for all practical purposes, strain (and strain hardening) may be considered to be uniform. However, as the volume deformed approaches the small volume associated with an imperfection, the material shows obvious signs of the basic inhomogeneous character of strain. The mean flow stress rises and the ends of the active shear planes are evident in a free surface as in figure 5. This is called the size effect.

In metal cutting, the undeformed chip thickness $(t)$ is small, and the width of shear zone $(\Delta y)$ is very small but the width of cut $(b)$ is relatively large. It would thus appear that the volume deformed at one time would be $(b t \Delta y / \sin \phi)$. However, when the back of a continuous chip is observed under the microscope it is found (figure 5) that the edges of the slip bands that are observed are not continuous across the width of the chip but have an extent characteristic of the imperfection spacing $(a)$. Thus, the volume deformed at any one time should be taken to $(b t \Delta y / \sin \phi)$ where $a \ll b$. In metal cutting, this volume appoachs $a^{3}$ (mean imperfection volume) and there is a size effect. This is the main reason specific energy $(u)$ increases with decrease in undeformed chip thickness $(t)$.

Under ordinary conditions, the shear planes are very closely spaced corresponding to the closeness of spacing of the weak spots in the metal. It may be assumed that slip planes are so spaced that a single weak spot is present on each plane. Drucker (1949) has employed a random array of weak points to qualitatively demonstrate the increase in unit cutting energy with decrease in depth of cut. However, in as much as the spacing of weak points is very small compared with the usual depths of cut, an orderly array of weak spots seems justified. The dots shown in figure 26 represent such an orderly arrangement of weak points to an exaggerated scale. These points have a uniform spacing of a unit in each direction.

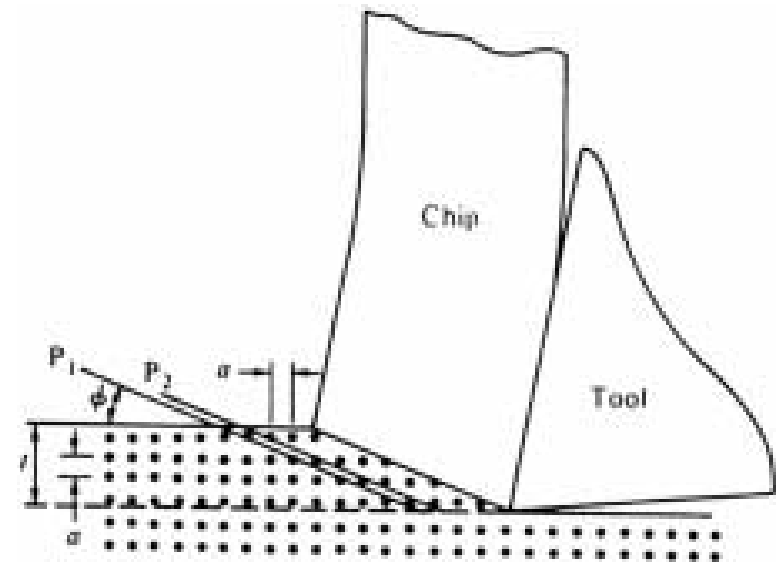

Figure 26. A specimen with uniform distribution of points of stress concentration (weak points) (after Shaw 1950). 
Let $P_{1}$ and $P_{2}$ be two shear planes making an angle $\phi$ with the direction of cut and passing through adjacent points in the first row below the surface. If the depth of cut is $t$, then $t / a$ planes may be placed between those at $P_{1}$ and $P_{2}$ such that a single plane passes through each weak spot in the layer in the process of being cut. The number of planes per unit distance in a direction perpendicular to the shear plane will be

$$
n=t /[a(a \sin \phi)]
$$

or the spacing of successive planes is

$$
\Delta y=\left(a^{2} \sin \phi\right) / t
$$

The total slip on a given shear plane is

$$
x=\left[\left(z^{2} \sin \phi\right) / t\right] \gamma
$$

where $\gamma$ is the unit uniform strain.

Assuming normal stress on the shear plane that is sufficient to suppress microcrack formation as in the Langford \& Cohen experiments, the flow stress should vary with strain as given by (10). However, beyond this strain, (11) pertains giving rise to a source of displacement due to the formation, transport, and rewelding of microcracks in addition to strain due to dislocations.

\section{Alternative origin of the size effect}

It is appropriate at this point to mention that an alternative explanation for the increase in hardness that occurs when the indentation size is reduced in metals has recently been introduced (Stelmashenko et al 1993; Fleck et al 1994; Ma \& Clark 1995; Nix \& Gao 1998; Gao et al 1999). This is based on the fact that there is an increase in the strain gradient with reduction in indentation size. This has been extended by Dinesh et al (2001) to explain the size effect in machining. In the analysis by them the size effect in hardness is related to that in cutting, by assuming the von Mises criterion is applicable. Based on the experiments of Merchant considered in $\S 2$ it is evident that this is not applicable in steady state chip formation.

In this strain gradient theory two types of dislocations are proposed: geometrically necessary dislocations $\left(\rho_{g}\right)$ that are responsible for work hardening and statistically stored dislocations $\left(\rho_{S}\right)$ that are affected by a strain gradient. When $\rho_{g} \ll \rho_{S}$ conventional plasticity pertains (strain rate is unimportant) but when $\rho_{g} \ll \rho_{S}$, a constitutive equation including strain rate should be included.

The impression one obtains is that the strain gradient approach (Dinesh et al 2001) is uniquely responsible for the size effect in cutting. In their concluding remarks, it is suggested that it should be possible to verify the validity of the strain rate formulation by experiments designed to test predictions of this approach. This has not yet been done and until it is it will not be possible to determine whether the influence of strain rate is significant in the chip formation application. In any case, it is believed the explanation presented here based on the influence of defects and normal stress on the shear plane is sufficiently well-supported by the experiments described in $\S 3$ that it should not be considered insignificant. 


\section{References*}

Anderson T L 1991 Fracture mechanics (Boca Raton, FL: CRC Press)

Argon A S, Im J, Safoglu R 1975 Metall. Trans. A6: 825

Backer W R, Marshall E R, Shaw M C 1952 Trans. ASME. 74: 61

Barrett C S 1943 Structure of metals (New York: McGraw Hill) pp 295

Blazynski T Z, Cole J M 1960 Proc. Inst. of Mech. Eng. 174: 757

Bridgman P W 1952 Studies in large plastic flow and fracture (New York: McGraw Hill)

Dinesh D, Swaminathan S, Chandrasekar S, Farris T N 2001 Proc. ASME-Int. Mech. Eng. Conf. pp 1-8

Drucker D C 1949 J. AppI. Phys. 20: 1

Ernst H J, Merchant M E 1941 Trans. Am. Soc. Metals 29: 299

Eugene F 1952 Ann. CIRP 52(1): 13-17

Eyring H, Ree T 1961 Proc. Nat. Acad. Sci. 47: 526-537

Eyring H, Jhon M S 1969 Significant theory of liquids (New York: J Wiley and Sons)

Eyring H, Ree T, Harai N 1958 Proc. Natl. Acad. Sci. 44: 683

Fleck N A, Muller G M, Ashby M F, Hutchinson J M 1994 Acta Metall. Mater. 41: 2855

Gao H, Huang Y, Nix W D, Hutchinson J W 1999 J. Mech. Phys. Solids 47: 1239

Heidenreich R O, Shockley W 1948 Report on strength of solids (London: Phys. Soc.) p. 57

Kececioglu D 1958 Trans. ASME 80:149-168, 541-546

Kececioglu D 1960 Trans. ASME-J. Eng. Ind. 82: 79-86

Komanduri R, Brown R H 1967 Metals Mater. 95: 308

Kwon K B, Cho D W, Lee S J, Chu C N 1999 Ann. CIRP 47: 43-46

Langford G, Cohen M 1969 Trans. Am. Soc. Metals 62: 623

Merchant M E 1945 J. AppI. Phys. 16: (a) 267-275, (b) 318-324

Merchant M E 1950 Machining theory and practice (Am. Soc. Metals) pp 5-44

Ma Q, Clarke D R 1995 J. Mater. Res. 46: 477

Nix W D, Gao H 1998 J. Mech. Phys. Solids 10: 853

Taylor G I 1934 Proc. R. Soc. A145: 362

Piispanen V 1937 Teknillinen Aikakaushehti (Finland) 27: 315

Piispanen V 1948 J. Appl. Phys. 19: 876

Polanyi M 1934 Z. Phys. 89: 660

Shaw M C 1950 J. Appl. Phys. 21: 599

Shaw M C 1952 J. Franklin Inst. 254/2: 109

Shaw M C 1980 Int. J. Mech. Sci. 22: 673-686

Stelmashenko N A, Walls M G, Brown L M, Milman Y V 1993 Acta. Metall. Mater. 41: 2855

Taniguchi N 1994 Precision Eng. 16: 5-24

Orowan E 1934 Fatigue and fracture of metals (New York: John Wiley \& Sons)

Usui E, Gujral A, Shaw M C Int. J. Machine Tools Res. 1: 187-197

Vyas A, Shaw M C 1999 Trans. ASME J. Mech. Sci. 121:163-172

Walker T J $1967 \mathrm{PhD}$ dissertation, Carnegie-Mellon University, Pittsburg, PA

Walker T J, Shaw M C 1969 Advances in machine tool design and research (Oxford: Pergamon) pp 241-252

Zhang B, Bagchi A 1994 Trans. ASME- J. Eng. Ind. 116: 289

\footnotetext{
*References in this list are not in journal format
} 